\title{
Elastic scattering of Beryllium isotopes near the Coulomb barrier
}

Cite as: AIP Conference Proceedings 1377, 144 (2011); https://doi.org/10.1063/1.3628371

Published Online: 31 October 2011

A. Di Pietro, F. Amorini, P. Figuera, M. Fisichella, M. Lattuada, A. Musumarra, M. Papa, M. G. Pellegriti, G. Randisi, F. Rizzo, D. Santonocito, G. Scalia, V. Scuderi, E. Strano, D. Torresi, L. Acosta, I. Martel, F. PerezBernal, M. J. G. Borge, O. Tengblad, A. Maira Vidal, L. M. Fraile, H. Jeppesen, D. Voulot, F. Wenander, J. Gomez-Camacho, M. Milin, R. Raabe, and M. Zadro

\section{ARTICLES YOU MAY BE INTERESTED IN}

The ${ }^{237} \mathrm{~Np}(\mathrm{n}, \mathrm{f})$ cross section at the CERN n-TOF facility

AIP Conference Proceedings 1377, 459 (2011); https://doi.org/10.1063/1.3628445
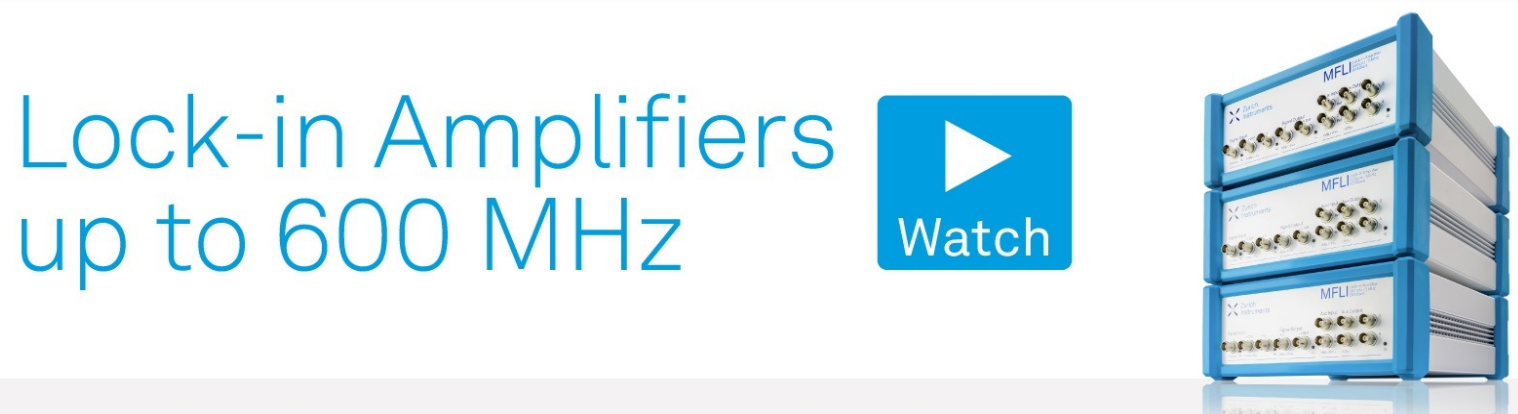


\title{
Elastic scattering of Beryllium isotopes near the Coulomb barrier.
}

\author{
A. Di Pietro*, F. Amorini ${ }^{\dagger, *}$, P. Figuera*, M. Fisichella ${ }^{\dagger, *}$, M. Lattuada ${ }^{\dagger, *}$, \\ A. Musumarra ${ }^{\dagger, *}$, M. Papa ${ }^{* *}$, M.G. Pellegriti ${ }^{\dagger, *}$, G. Randisi $i^{\dagger, *}$, F. Rizzo ${ }^{\dagger, *}$, \\ D. Santonocito ${ }^{\dagger, *}$, G. Scalia ${ }^{\dagger, *}$, V. Scuderi ${ }^{\dagger, *}$, E. Strano ${ }^{\dagger, *}$, D. Torresi ${ }^{\dagger, *}$, \\ L. Acosta ${ }^{\ddagger}$, I. Martel ${ }^{\ddagger}$, F. Perez-Bernal ${ }^{\ddagger}$, M.J.G. Borge ${ }^{\S}$, O. Tengblad ${ }^{\S}$, \\ A. Maira Vidal ${ }^{\S}$, L.M. Fraile II $^{\text {, H. Jeppesen }}{ }^{\text {II }}$, D. Voulot ${ }^{\text {II }}$, F. Wenander ${ }^{\text {II }}$, \\ J. Gomez-Camacho", M. Milin ${ }^{\dagger \dagger}$, R. Raabe ${ }^{\ddagger \ddagger}$ and M. Zadro ${ }^{\S \S}$ \\ ${ }^{*}$ INFN-Laboratori Nazionali del Sud, Catania, Italy \\ ${ }^{\dagger}$ Dipartimento di Fisica ed Astronomia Universitá di Catania, Catania, Italy \\ ** INFN-Sezione di Catania, Catania, Italy \\ ¥Departamento de Fisica Aplicada Universidad de Huelva, Huelva, Spain \\ ${ }^{\S}$ Instituto de Estructura de la Materia CSIC, Madrid, Spain \\ IISOLDE, CERN, CH-1211 Geneva 23, Switzerland \\ "Departamento de Fisica Atomica Molecular Nuclear Universidad de Sevilla, and Centro \\ Nacional de Aceleradores, Sevilla, Spain \\ ${ }^{\dagger}$ Department of Physics Faculty of Science University of Zagreb, Zagreb, Croatia \\ $\$$ Instituut voor Kern-en Stralingsfysica K.U.Leuven, Belgium \\ ${ }_{\S}$ Division of Experimental Physics Ruđer Bošković Institute, Zagreb, Croatia
}

\begin{abstract}
In this contribution, results of experiments performed with the three Beryllium isotopes ${ }_{9,10,11} \mathrm{Be}$ on a medium mass ${ }^{64} \mathrm{Zn}$ target, at a center of mass energy of $\approx 1.4$ the Coulomb barrier, will be discussed. Elastic scattering angular distributions have been measured for the ${ }^{9,10} \mathrm{Be}$ reactions. In the ${ }^{11} \mathrm{Be}$ case the quasielastic scattering angular distribution was obtained. In the halo nucleus case, the angular distribution exhibit a non-Fresnel-type pattern with a strong damping of the Coulombnuclear interference peak. Moreover, it is found that the total reaction cross-section for the halo nucleus induced collision is more than double the ones extracted in the collisions induced by the non-halo Beryllium isotopes. A large contribution to the total-reaction cross-section in the ${ }^{11} \mathrm{Be}$ case could be attributed to transfer and/or break-up events.
\end{abstract}

Keywords: halo nuclei, elastic scattering, total reaction cross-section, direct reactions PACS: $25.60 . \mathrm{Bx}, 25.70 . \mathrm{Bc}$

\section{INTRODUCTION}

Since the discovery of a phenomenon called "nuclear halo" [1], several reaction studies have been performed with halo nuclei in the attempt to understand their peculiar structure. The single-particle wave function of these nuclei has a long tail, extending mostly outside the potential well. Elastic scattering, being a peripheral process, is an ideal tool to probe the tail of the wave function and hence to learn about the surface properties of halo nuclei. Low energy elastic scattering experiments, involving halo nuclei, have mostly been performed with the $2 \mathrm{n}$-halo ${ }^{6} \mathrm{He}$ nucleus on several targets over a wide range of masses. Such studies have shown that coupling to the continuum (break-up), strongly affects the elastic cross-section. On heavy targets the elastic scattering angular 
distribution results suppressed at forward angles, in the Coulomb-nuclear interference region. This suppression is partially attributed to the coupling to the Coulomb dipole excitation of the low-lying E1 strength [2]. Due to the large spatial extension of halo nuclei, reactions initiated by such nuclei exhibit large total-reaction cross-sections see e.g. [3, 4]. At low energies, near the Coulomb barrier, direct processes such as transfer and break-up are mostly contributing to this cross-section. Exclusive measurements have shown that, among these processes, the $2 \mathrm{n}$ transfer gives the largest contribution $[5,6]$. In the reaction induced by the $1 \mathrm{n}$-halo ${ }^{11} \mathrm{Be}$ nucleus on a ${ }^{209} \mathrm{Bi}$ target at energy near the Coulomb barrier, the extracted total-reaction cross-section was found to be similar to the one of ${ }^{9} \mathrm{Be}+{ }^{209} \mathrm{Bi}[7,8]$, measured by the same group [9]. Since the absorption is originated by fusion, which for the two reactions ${ }^{9,11} \mathrm{Be}+{ }^{209} \mathrm{Bi}$ has a similar cross-section [10] and break-up, the authors concluded that the breakup process must have comparable strengths in both ${ }^{9,11}$ Be nuclei.

In this contribution new results of experiments performed with the three Beryllium isotopes ${ }^{9,110,11} \mathrm{Be}$ on a medium mass ${ }^{64} \mathrm{Zn}$ target will be discussed.

\section{EXPERIMENTS}

The experiment with the stable weakly-bound ${ }^{9} \mathrm{Be}$ beam was performed at Laboratori Nazionali del Sud (LNS) in Catania using the beam delivered by the 14MV SMP Tandem of LNS. The energy of the beam was $\mathrm{E}_{\mathrm{c} . \mathrm{m} .}=24.9 \mathrm{MeV}$. The ${ }^{9} \mathrm{Be}$ was impinging on a $550 \mu \mathrm{g} / \mathrm{cm}^{2}{ }^{64} \mathrm{Zn}$ target. The elastic scattering angular distribution was measured by using five collimated surface barrier Si detector telescopes $(10 \mu \mathrm{m} \Delta E$ and $200 \mu \mathrm{m} E$ detectors), placed on a rotating platform. By rotating the platform, the elastic-scattering angular distribution was obtained up to $\theta_{\text {c.m. }}=110^{\circ}$. The experiment with the radioactive ${ }^{10,11} \mathrm{Be}$ beams, was performed at Rex-ISOLDE (CERN). The beam energy was $\mathrm{E}_{\mathrm{c.m} .}=24.5 \mathrm{MeV}$ for both beams. In order to compensate for the low intensity of the radioactive beams (the average beam intensity was $10^{6}$ and $10^{4}$ pps for ${ }^{10} \mathrm{Be}$ and ${ }^{11} \mathrm{Be}$ respectively), a large solid-angle detection system was used. The detectors, consisting of an array of six Si-detector-telescopes each formed by a $40 \mu \mathrm{m}, 50 \times 50 \mathrm{~mm}^{2}, \Delta E$ DSSSD detector (16+16 strips) and a $1500 \mu \mathrm{m}$ single pad $E$ detector, were placed very close to the target in order to have a large angular $\left(10^{0} \leq \theta \leq 150^{\circ}\right)$ and solid angle coverage. A $550 \mu \mathrm{g} / \mathrm{cm}^{2}$ and $1000 \mu \mathrm{g} / \mathrm{cm}^{2}{ }^{64} \mathrm{Zn}$ target was used with ${ }^{10} \mathrm{Be}$ and ${ }^{11} \mathrm{Be}$ beams respectively. In order to detect particles at $\theta \approx 90^{\circ}$, the target was angled at $45^{\circ}$. Since the detectors were placed very close to the target, small shifts of the beam axis resulted in a non negligible variation of the detector angles. In the off-line analysis information on the beam shift on the target were obtained by looking at the small angle Rutherford scattering in the two front detectors placed symmetrically with respect to the beam axis. In order to check the adopted procedure, ${ }^{12} \mathrm{C},{ }^{10} \mathrm{Be}+{ }^{197} \mathrm{Au}$ Rutherford scattering at energies $E_{\mathrm{cm}}=25.7$ and $27.9 \mathrm{MeV}$ respectively was also measured. In Fig. 1 the experimental set-up for the ${ }^{10,11} \mathrm{Be}+{ }^{64} \mathrm{Zn}$ is shown.

Due to the energy resolution of the radioactive beams $(\approx 1 \%)$, in addition to the energy staggling in the target, it was not possible to separate the ground state from the $\frac{1}{2}^{-}\left(E_{\mathrm{x}}=0.32 \mathrm{MeV}\right)$ state in ${ }^{11} \mathrm{Be}$. Therefore, in the ${ }^{11} \mathrm{Be}$ case, not the elastic but the quasielastic scattering is measured. 


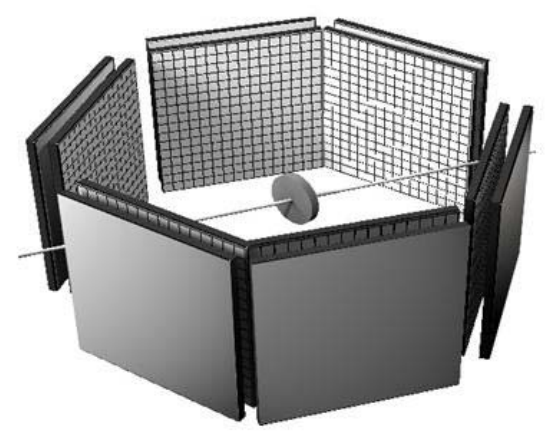

FIGURE 1. The set-up used for the experiment ${ }^{10,11} \mathrm{Be}+{ }^{64} \mathrm{Zn}$ at Rex-ISOLDE.

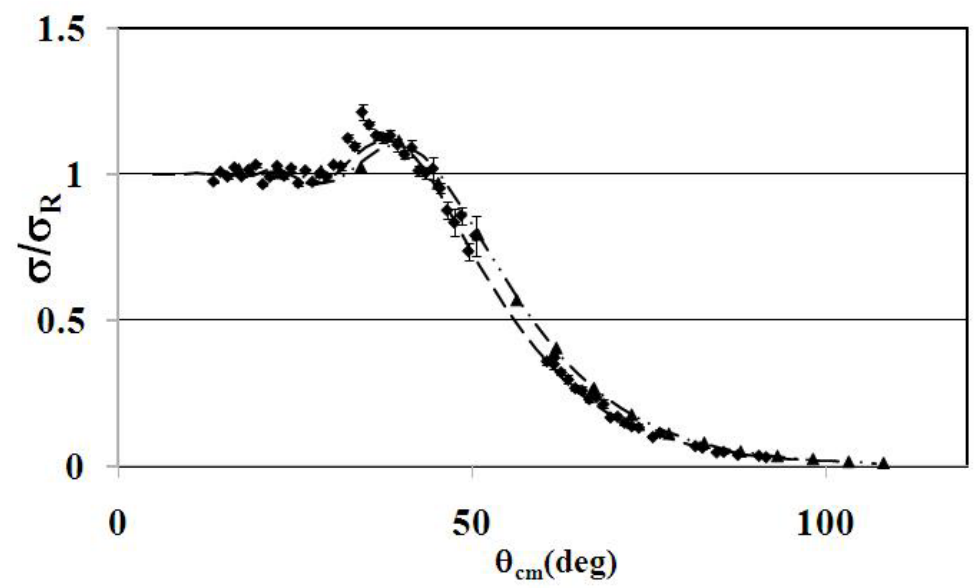

FIGURE 2. Elastic scattering angular distribution. Triangles ${ }^{9} \mathrm{Be}+{ }^{64} \mathrm{Zn}$. Diamonds ${ }^{10} \mathrm{Be}+{ }^{64} \mathrm{Zn}$. Lines represent the result of the Optical Model fit to the data (see text for details).

\section{EXPERIMENTAL RESULTS AND DISCUSSION.}

In Fig. 2 it is shown a comparison of the extracted elastic scattering angular distribution for ${ }^{9,10} \mathrm{Be}+{ }^{64} \mathrm{Zn}$. The ${ }^{9} \mathrm{Be}$ is a stable weakly-bound nucleus $\left(\mathrm{S}_{n}=1.67 \mathrm{MeV}\right)$, whereas ${ }^{10} \mathrm{Be}$ is radioactive but well bound $\left(\mathrm{S}_{n}=6.81 \mathrm{MeV}\right)$. As one can see from the figure, the two angular distributions appear to be very similar. This result is not surprising, ${ }^{9} \mathrm{Be}$ and ${ }^{10} \mathrm{Be}$ have similar radii, are equally deformed and with a well developed cluster structure. Moreover, at energies close to the Coulomb barrier, the reaction cross-section in the ${ }^{9} \mathrm{Be}+{ }^{64} \mathrm{Zn}$ case is fully saturated by the total-fusion cross-section and the break-up of the weakly bound ${ }^{9} \mathrm{Be}$ is not giving an important contribution [11]. 


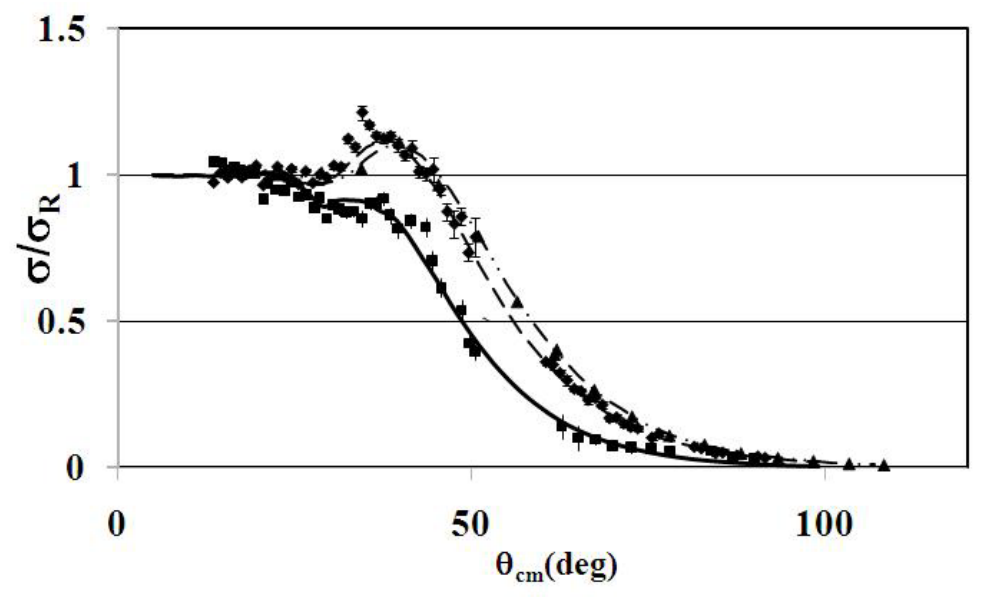

FIGURE 3. Elastic scattering angular distribution for ${ }^{9} \mathrm{Be}+{ }^{64} \mathrm{Zn}$ triangles and ${ }^{10} \mathrm{Be}+{ }^{64} \mathrm{Zn}$, diamonds. quasielastic angular distribution for ${ }^{11} \mathrm{Be}+{ }^{64} \mathrm{Zn}$, squares. Lines represent the result of the Optical Model fit to the data (see text for details).

In Fig. 3 the quasielastic scattering angular distribution of ${ }^{11} \mathrm{Be}+{ }^{64} \mathrm{Zn}$ is shown together with the ${ }^{9,10} \mathrm{Be}+{ }^{64} \mathrm{Zn}$ elastic scattering angular distribution. The ${ }^{11} \mathrm{Be}+{ }^{64} \mathrm{Zn}$ angular distribution shows a very different behaviour. The peak due to the interference between the Coulomb and nuclear amplitude is missing and the quasielastic crosssection appear to be suppressed at all angles at which the nuclear interaction is felt $\left(\sigma_{\text {ela }} / \sigma_{\text {Ruth }}<1\right)$.

Angular distributions having similar shapes of the ${ }^{11} \mathrm{Be}+{ }^{64} \mathrm{Zn}$ one have been observed when a strong coupling with the Coulomb excitation of quadrupole states in heavy deformed nuclei is present [12]. The suppression of the elastic cross-section is due to absorption occurring at large distances owing to the long-range Coulomb interaction.

In the ${ }^{11} \mathrm{Be}$ case, absorption at large distances may occur due to the large radial extension of the halo nucleus. In [13] the suppression of the elastic cross-section in the Coulomb-nuclear interference region is studied as a function of the target charge and beam energy. According to [13], in the case of scattering of a halo nucleus with a lowcharge target at energy near the barrier, the major contribution to the long range absorption cannot be attributed to a strong Coulomb dipole coupling due to the presence of the low-lying dipole strength near the threshold. Coupling with the Coulomb dipole low-lying strength is expected to be important in the scattering with high charge targets, as experimentally observed in collisions induced by the 2 n-halo ${ }^{6} \mathrm{He}$ nucleus [2]. In the scattering of ${ }^{11} \mathrm{Be}$, investigated in the present case, nuclear effects are important and cannot be neglected. 


\section{Optical Model analysis.}

We analyzed the ${ }^{9,10,11} \mathrm{Be}+{ }^{64} \mathrm{Zn}$ scattering angular distribution using the Optical Model (OM). For the ${ }^{9,10} \mathrm{Be}+{ }^{64} \mathrm{Zn}$ elastic scattering, a volume potential having a WoodsSaxon (W-S) shape for both the real and imaginary part was used. In the case of ${ }^{9,10} \mathrm{Be}$, the fit procedure was the following: the radius and diffuseness (real and imaginary) were fixed and the best $\chi^{2}$ was obtained by varying the potential depths. Before fixing radius and diffuseness several calculation were performed where these were varied at steps of $0.05 \mathrm{fm}$. Using the above mentioned procedure, the final fit was performed with a reduced number of free parameters. The fit was done using the code PTOLEMY [14] and the results are shown in Fig. 2 and 3. In the case of the quasielastic scattering angular distribution of ${ }^{11} \mathrm{Be}+{ }^{64} \mathrm{Zn}$, in order to take the coupling to the break-up into consideration, a Dynamic Polarization Potential (DPP) was considered. The DPP used is an imaginary surface potential having the shape of a W-S derivative. No real part for the DPP potential was considered. Moreover, in the ${ }^{11} \mathrm{Be}$ case, the volume potential responsible for the core-target interaction, is the one extracted from the ${ }^{10} \mathrm{Be}+{ }^{64} \mathrm{Zn}$ elastic scattering fit. The OM fit was performed using as free parameter the depth of the DPP potential and varying the diffuseness at steps of $0.05 \mathrm{fm}$. The best $\chi^{2}$ were obtained for a DPP diffuseness parameter of $\mathrm{a}_{s i} \approx 3.5 \mathrm{fm}$. The results are shown in Fig. 3. The values of the obtained potential parameters can be found in [15]. This large diffuseness is necessary in order to reproduce the suppression of the quasielastic cross-section in the Coulomb-nuclear interference region. The addition of an attractive surface potential is hence responsible for the increase of the reaction cross-section due to break-up/transfer of the halo ${ }^{11} \mathrm{Be}$ nucleus.The obtained result is in agreement with the calculations of [16] and [17] where a surface diffuseness of $3.2 \mathrm{fm}$ of the DPP potential was obtained. As mentioned above, in the ${ }^{11} \mathrm{Be}+{ }^{64} \mathrm{Zn}$ case, the inelastic excitation of the ${ }^{11} \mathrm{Be}$ at $\mathrm{E}_{x}=0.32 \mathrm{MeV}$ could not be separated from the elastic and therefore the quasielastic scattering angular distribution was obtained. In order to evaluate the contribution due to the inelastic scattering to the quasielastic angular distribution, DWBA calculations were performed. In these calculations we used as nuclear form factor the radial derivative of the real and imaginary OP obtained from the scattering data and as dipole strength $\mathrm{B}(E 1)=0.115 e^{2} \mathrm{fm}^{2}$ [18]. These calculations show that the inelastic contribution to the quasielastic scattering angular distribution is small and hence the quasielastic angular distribution can be regarded as elastic scattering angular distribution. This can be seen in Fig. 4. The total-reaction cross-sections deduced from OM analysis are $\sigma_{R}=1090 \mathrm{mb}$ for ${ }^{9} \mathrm{Be}, \sigma_{R}=1260 \mathrm{mb}$ for ${ }^{10} \mathrm{Be}$ and $\sigma_{R}=2730 \mathrm{mb}$ for ${ }^{11} \mathrm{Be}$.

\section{Cross-section for direct reactions}

The total-reaction cross-section in the ${ }^{11} \mathrm{Be}+{ }^{64} \mathrm{Zn}$ case is $\approx 2.2$ times larger than the total-reaction cross-section of ${ }^{9,10} \mathrm{Be}+{ }^{64} \mathrm{Zn}$ collisions. The question that arises is: which are the processes contributing to this large cross-section? In the ${ }^{11} \mathrm{Be}+{ }^{64} \mathrm{Zn}$ experiment an attempt to measure the fusion cross-section was made but unfortunately the beam intensity was too small and no information on this cross-section were obtained. 


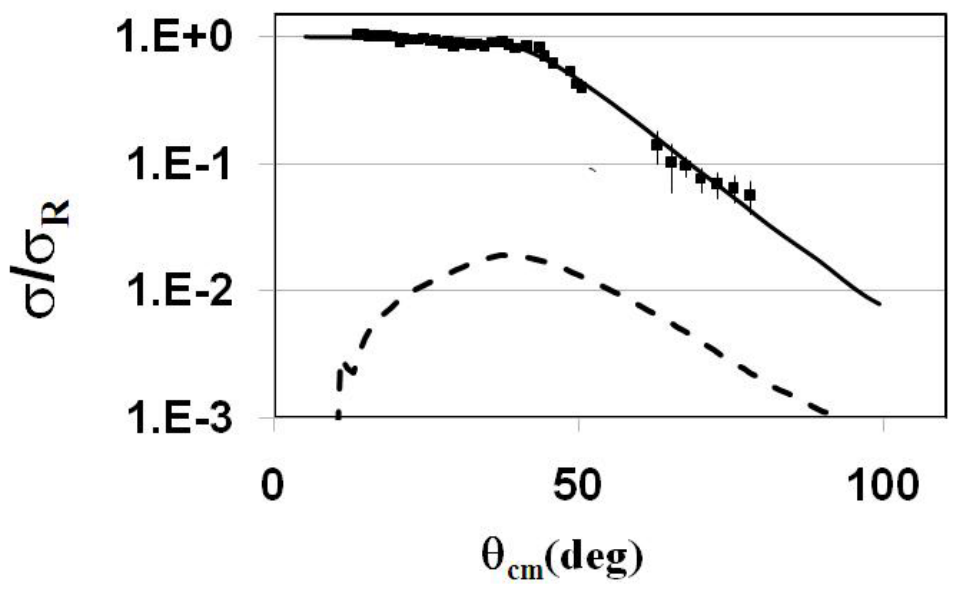

FIGURE 4. quasielastic angular distribution for ${ }^{11} \mathrm{Be}+{ }^{64} \mathrm{Zn}$, squares. Full line represent the result of the Optical Model fit to the data. Dashed line represent DWBA calculations of inelastic scattering. See text for details.

From the analysis of the $\Delta E \% E$ spectra in the ${ }^{11} \mathrm{Be}+{ }^{64} \mathrm{Zn}$ case, events next to the ${ }^{11} \mathrm{Be}$ quas-elastic peak were observed. The position of those events in the $\Delta E \% E$ plot was consistent with the detection of ${ }^{10} \mathrm{Be}$ coming from transfer and/or break-up processes; such events are not observed in the ${ }^{10} \mathrm{Be}$ beam case. In Fig. 5 the $\Delta E \% E$ spectrum at $\theta=35^{\circ}$ is shown. For the above mentioned events the angular distribution was obtained and it is shown in Fig. 6. The integrated cross-section for transfer/break-up reaction is $\sigma=1100 \pm 150 \mathrm{mb}$, corresponding to $40 \%$ of the exstracted total-reacion cross-section. It is found that, contrary to the weakly bound ${ }^{9} \mathrm{Be}$ case, where at a similar $\mathrm{E}_{\mathrm{c} . \mathrm{m} \text {. }}$ energy, the total-reaction cross-section is mainly due to fusion [11], in the ${ }^{11} \mathrm{Be}$ case direct processes are giving a large contribution to this cross-section.

\section{CONCLUSION}

The scattering angular distribution for the reactions ${ }^{9,10,11} \mathrm{Be}+{ }^{64} \mathrm{Zn}$ have been measured at $\approx 1.4$ the Coulomb barrier energy. Contrary to the elastic-scattering angular distribution for ${ }^{9,10} \mathrm{Be}+{ }^{64} \mathrm{Zn}$ which shows the typical Fresnel-type scattering shape, this feature is not observed in the ${ }^{11} \mathrm{Be}$ quasielastic scattering. The peak due to Coulomb-nuclear interference, is missing and absorption occurs at much longer distances than for the other two Be isotopes [15]. In the OM analysis, in order to reproduce the ${ }^{11} \mathrm{Be}$ data, a surface DPP potential was used in addition to the volume potential. This last potential was extracted from the ${ }^{10} \mathrm{Be}+{ }^{64} \mathrm{Zn}$ elastic scattering $\mathrm{OM}$ fit. The best fit was obtained with the surface term having a very large diffuseness $a_{\mathrm{si}} \approx 3.5 \mathrm{fm}$ in agreement with what found by $[16,17]$. The total-reaction cross-section was extracted from the OM fit. A much larger cross-section is obtained in the ${ }^{11} \mathrm{Be}$ case. Responsible for this large cross-section are partially direct reaction processes such as transfer and break-up. These 


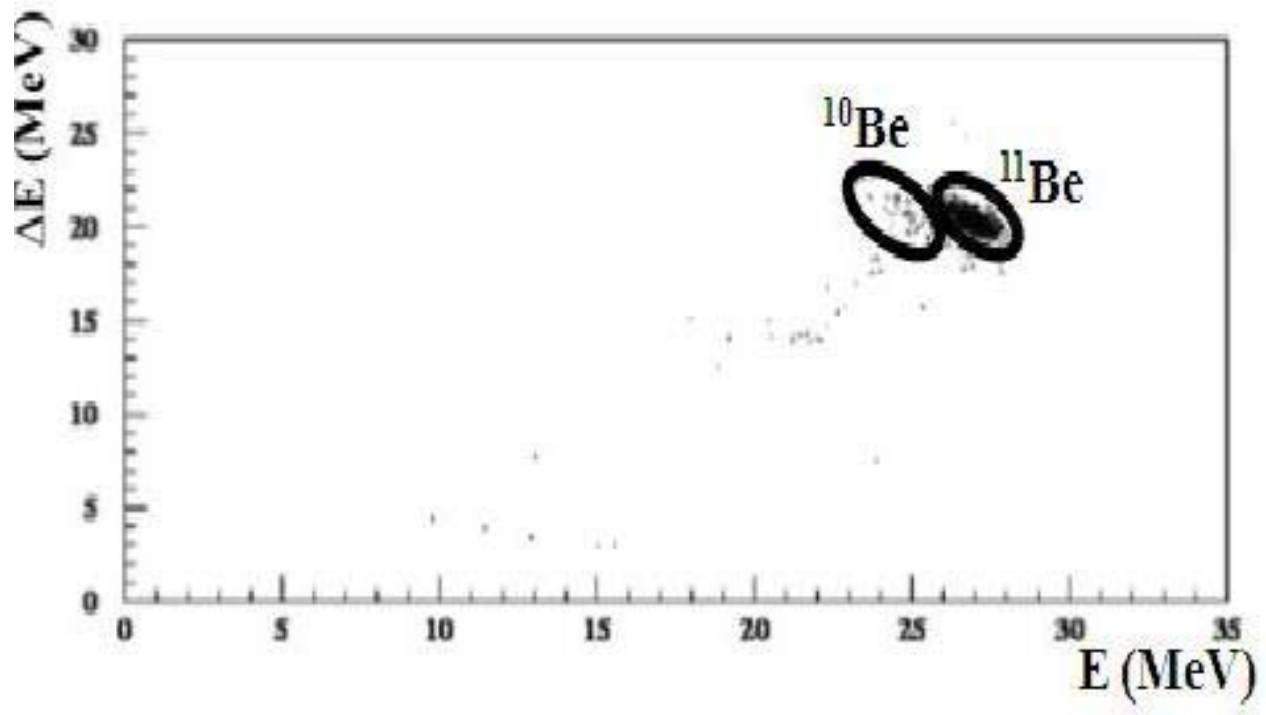

FIGURE 5. $\Delta E \% E$ scatter plot for the reaction ${ }^{11} \mathrm{Be}+{ }^{64} \mathrm{Zn}$.

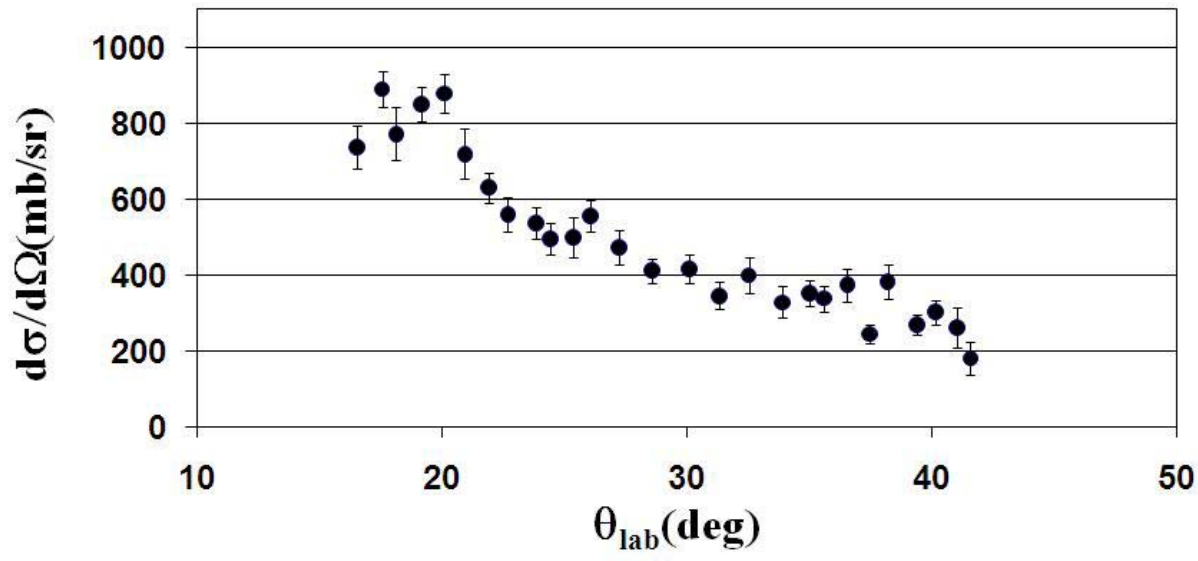

FIGURE 6. Angular distribution of transfer/break-up events in ${ }^{11} \mathrm{Be}+{ }^{64} \mathrm{Zn}$ obtained by selecting ${ }^{1} 0 \mathrm{Be}$ events in the $\Delta E \% E$ spectrum.

processes, instead, do not play an important role in the weakly-bound ${ }^{9} \mathrm{Be}$ induced collision where the total reaction cross-section is found to be similar to the well bound ${ }^{10} \mathrm{Be}$ case. A behaviour of the elastic angular distribution as the one observed in the ${ }^{11} \mathrm{Be}$ case is found in collision induced by the $2 \mathrm{n}$-halo ${ }^{6} \mathrm{He}$ nucleus on heavy targets where coupling with the Coulomb dipole break-up is important. However, the large suppression of the elastic cross-section in the Coulomb-nuclear interference region 
has not been observed in the ${ }^{6} \mathrm{He}+{ }^{64} \mathrm{Zn}$ [3]. The larger radial distribution of the ${ }^{11} \mathrm{Be}$ compared to the ${ }^{6} \mathrm{He}[17]$ could be responsible for such a difference.

\section{REFERENCES}

1. P.G. Hansen, A.S. Jensen and B. Jonson, Ann. Rev. Nucl. Part. Sci. 45 (1995) 591 and ref. therein.

2. A. M. Sánchez-Benítez et al., Nucl. Phys. A 803, 30 (2008).

3. A. Di Pietro et al., Phys. Rev. C 69, 044613 (2004).

4. E.F. Aguilera et al., Phys. Rev. C 63,061603R (2001).

5. A. Chatterjee et al., Phys. Rev. Lett. 101, 032701 (2008).

6. P. A. DeYoung et al., Phys. Rev. C 71, 051601(R) (2005).

7. M. Mazzocco et al., Eur. Phys. J. A 28, 295 (2006).

8. M. Mazzocco et al., Eur. Phys. J. S. T. 150, 37 (2007).

9. C. Signorini et al., Nucl. Phys. A 701, 23c (2002).

10. P. A. DeYoung et al., Eur. Phys. J. A 2, 27 (1998).

11. S.B. Moraes et al., Phys. Rev. C 61, 064608 (2000).

12. W.G. Love, T. Teresawa and G.R. Satchler, Nucl. Phys. A 291, 183 (1977).

13. Y. Kucuk, I. Boztosun and N. Keeley, Phys. Rev. C 79, 067601 (2009).

14. M.J. Rhoades-Brown et al., Phys. Rev. C 21, 2417 (1980).

15. A. Di Pietro et al., Phys. Rev. Lett. 105, 022701 (2010).

16. A. Bonaccorso and F. Carstoiu, Nucl. Phys. A 706, 322 (2002).

17. M. Hassan et al., Phys. Rev. C 79, 064608 (2009).

18. F. Ajzenberg-Selove, Nucl. Phys. A 506, 1 (1998). 